\title{
Simulation of ${ }^{99}$ Mo Production from $30 \mathrm{MeV}$ Electron Linear Accelerator
}

\author{
T. ZAWISTOWSKI* AND S. WRONKA \\ National Centre for Nuclear Research, A. Soltana 7, Otwock-Świerk 05-400, Poland \\ Doi: 10.12693/APhysPolA.139.451 \\ *e-mail: tobiasz.zawistowski@ncbj.gov.pl

\begin{abstract}
The purpose of the research was to simulate the process of ${ }^{99} \mathrm{Mo}$ production. The most widely used isotope for medical imaging is ${ }^{99 m}$ Tc. This radionuclide is produced via ${ }^{99}$ Mo beta minus decay. A relatively new option is a linac-based way of obtaining ${ }^{99}$ Mo through a photonuclear reaction ${ }^{100} \mathrm{Mo}(\gamma, \mathrm{n})^{99} \mathrm{Mo}$ with bremsstrahlung irradiation. The purpose of this study was to simulate the process of ${ }^{99}$ Mo production using a $30 \mathrm{MeV}$ electron linear accelerator with a $100 \mu \mathrm{A}$ average beam current. The Monte Carlo calculations were used for this purpose. The main goal of the presented work was to optimize the process of irradiation of the ${ }^{100} \mathrm{Mo}$ target with an electron beam in order to produce ${ }^{99} \mathrm{Mo}$ with the highest efficiency. Parameters like FWHM of the beam and thickness of conversion target were investigated. The simulation was carried out using the Monte Carlo method with FLUKA 2011.2x.4 and Flair_2.3-0_all. The study showed that the highest efficiency (photons production) among widely used conversion targets is at an about $3 \mathrm{~mm}$ thick round tungsten plate. Results of the executed simulations showed that there is a possibility of obtaining $300 \mathrm{GBq}$ of ${ }^{99}$ Mo within three days of irradiation of a $99 \%$ enriched ${ }^{100}$ Mo target $(\varphi=3 \mathrm{~cm}, 5 \mathrm{~mm}$ thickness $)$.
\end{abstract}

topics: molybdenum-99, technetium-99m, irradiation, radioisotopes

\section{Introduction}

The most widely used isotope for medical imaging is ${ }^{99 m} \mathrm{Tc}$. This radionuclide is produced via ${ }^{99}$ Mo beta minus decay. A relatively new option is a linear accelerator-based production of ${ }^{99} \mathrm{Mo}$ through a photonuclear reaction ${ }^{100} \mathrm{Mo}(\gamma, \mathrm{n}){ }^{99} \mathrm{Mo}$ with bremsstrahlung irradiation. The purpose of this study was to simulate the process of ${ }^{99}$ Mo production using a $30 \mathrm{MeV}$ electron linear accelerator with $100 \mu \mathrm{A}$ average beam current. The Monte Carlo calculations were used for this purpose. The main goal of the presented work was to optimize the process of irradiation of a $99.9 \%$ enriched ${ }^{100} \mathrm{Mo}$ target with an electron beam in order to produce ${ }^{99} \mathrm{Mo}$ with the highest efficiency for the proposed target system.

\section{Methodology}

Simulations of a photon converter and photoneutron targets irradiation depending on their geometry and thickness were performed. Tungsten and tantalum were investigated as photon conversion targets. Dependence of FWHM of the beam on bremsstrahlung photons energy was studied. The simulations were carried out using the Monte Carlo method with the FLUKA 2011.2x.4 program [1]. The program code interface was Flair_2.3-0_all.
As the MC method requires high-efficiency processors, the Świerk Computing Centre (CIŚ - Centrum Informatyczne Świerk) hardware was applied for this purpose.

\section{Results}

It was found that FWHM as the electron beam parameter did not affect photons energy spectrum, therefore a (wider than optional $1 \mathrm{~mm}$ ) default $4 \mathrm{~mm}$ FWHM beam parameter was chosen. The assumed geometry and scheme of the target system are presented in Fig. 1. The gray rectangle (blue indicator on the left) means $3 \mathrm{~mm}$ of tungsten target and the green rectangle (blue indicator on the right) represents a $5 \mathrm{~mm}$ molybdenum target. Space "material" used in the simulation was pure air.

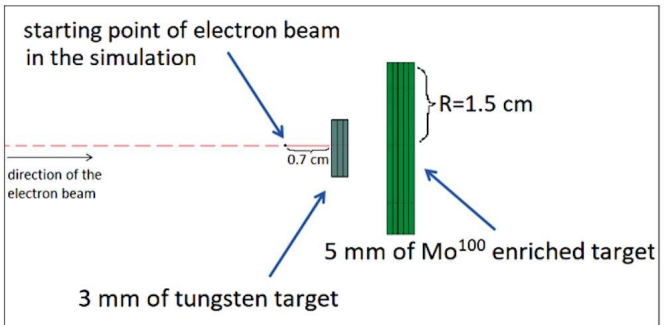

Fig. 1. Geometry and scheme of the targets system. 


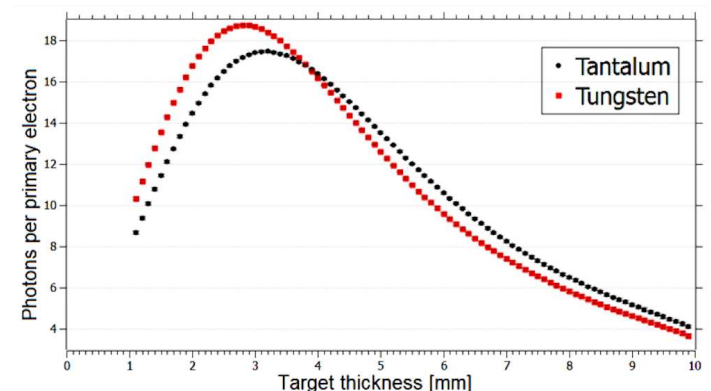

Fig. 2. Comparison of the conversional targets.

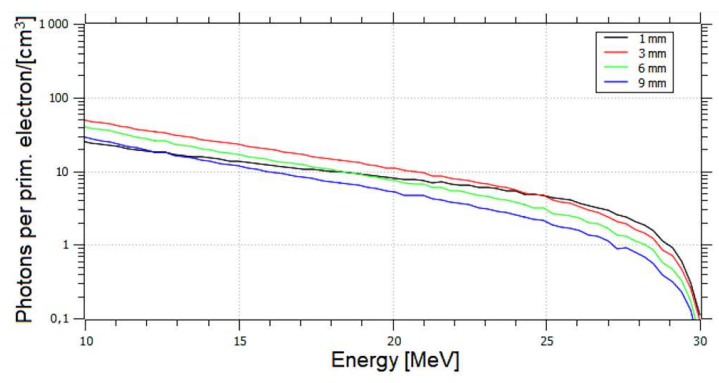

Fig. 3. Bremsstrahlung photons depending on tungsten thickness.

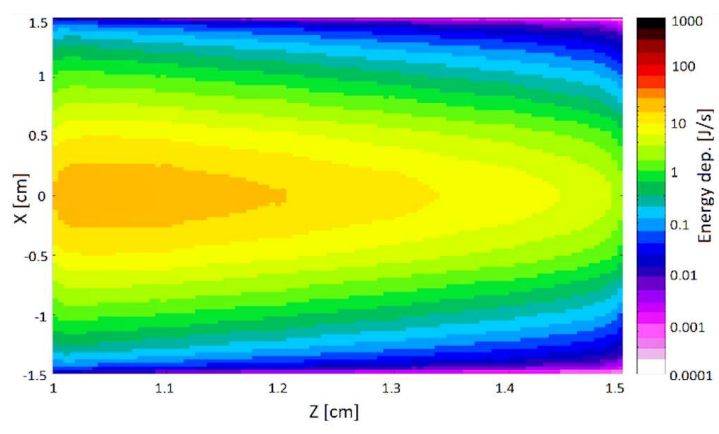

Fig. 4. Energy deposition in molybdenum target $(x-z$ plane $)$.

An important issue was the selection and optimization of the thickness of the conversion target. The comparison of two converters is shown in Fig. 2. The highest number of photons was obtained for an about $3 \mathrm{~mm}$ tungsten target. Maximum photon fluence can be obtained for a $3.2 \mathrm{~mm}$ tantalum target or a $2.8 \mathrm{~mm}$ tungsten target.

Tungsten is slightly more effective in generating bremsstrahlung photons when compared with tantalum. The photonuclear reaction $\left({ }^{100} \mathrm{Mo}(\gamma, \mathrm{n}){ }^{99} \mathrm{Mo}\right)$ needs photons with adequate energies. According to IAEA data, the threshold energy for ${ }^{100} \mathrm{Mo}(\gamma, \mathrm{n})^{99} \mathrm{Mo}$ photonuclear reaction is equal to $(8.2942 \pm 0.0004) \mathrm{MeV}[2,3]$. However, in some papers authors give a value of $9.8 \mathrm{MeV}[4,5]$ or $9 \mathrm{MeV}$ [6]. The measurement of the relative amount of high energy photons for different thicknesses of tungsten confirmed that the highest number of photons (with energies above threshold energy) could be gained for $3 \mathrm{~mm}$ thickness.

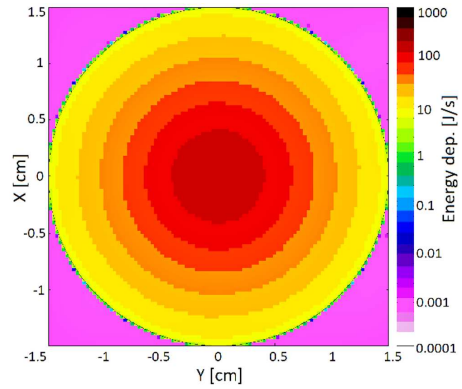

Fig. 5. Energy deposition in molybdenum target $(x-y$ plane $)$.

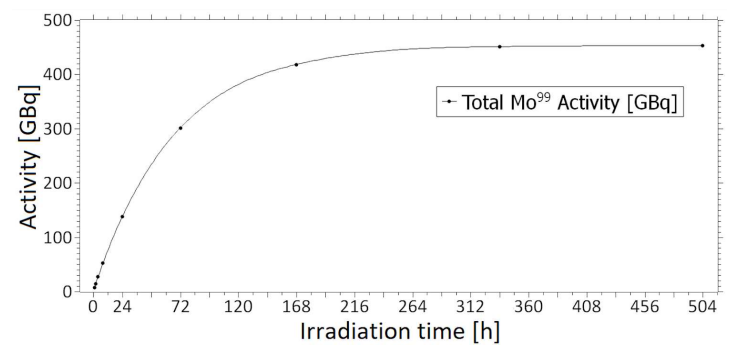

Fig. 6. Molybdenum-99 activity [GBq] depending on irradiation time.

The percentage share of the photons amount is different depending on converter thickness, but the highest amount of photons with desired energy is for the $3 \mathrm{~mm}$ tungsten conversion target. For clearer display results, the photons corresponding to the highest amount were marked by a red line (see Fig. 3).

The performed simulation included studies on energy deposition in the molybdenum target. It was found that during irradiation with photons the most exposed is the first $2 \mathrm{~mm}$ of the molybdenum target (Fig. 4). Energy deposition is concentric (Fig. 5) and the power equals about $500 \mathrm{~J} / \mathrm{s}$ in the middle of the target. This indicates that in the real experiment, a target cooling system seems to be necessary.

The activity dependence of ${ }^{99} \mathrm{Mo}$ produced with the $30 \mathrm{MeV}$ electron beam on ${ }^{100} \mathrm{Mo}$ target irradiation time is shown in Fig. 6.

It can be seen that as a result of $72 \mathrm{~h}$ irradiation of $30 \mathrm{~mm}$ in diameter and $5 \mathrm{~mm}$ thick ${ }^{100} \mathrm{Mo}$ target activity about $300 \mathrm{GBq}$ of ${ }^{99} \mathrm{Mo}$ will be produced. Such a great amount of ${ }^{99} \mathrm{Mo}$ nuclei could provide material for fifteen $20 \mathrm{GBq}$ molybdenum-technetium generators (for nuclear medicine). It could be an alternative way of obtaining ${ }^{99}$ Mo to the method of production using nuclear reactors.

\section{Conclusions}

The performed calculations showed that the $3 \mathrm{~mm}$ thick tungsten disc is the most optimal conversion target used for the production of ${ }^{99} \mathrm{Mo}$ via the nuclear reaction: ${ }^{100} \mathrm{Mo}(\gamma, \mathrm{n}){ }^{99} \mathrm{Mo}$. Using the 
conversion target, it is possible to obtain about $300 \mathrm{GBq}$ of ${ }^{99} \mathrm{Mo}$ after three days of irradiation of the ${ }^{100}$ Mo target ( $\varphi=30 \mathrm{~mm}$ and $5 \mathrm{~mm}$ thickness) with the $30 \mathrm{MeV}$ electron beam of $100 \mu \mathrm{A}$ average beam current. The obtained results will be verified by an experimental method in the near future.

\section{Acknowledgments}

The contribution of Tobiasz Zawistowski (RadFarm student) was realized within Project No. POWR.03.02.00-00-I009/17-00 (Operational Project Knowledge Education Development 20142020 co-financed by the European Social Fund).

\section{References}

[1] Fluka, User's Online Guide.

[2] T. Kawano, Y.S. Cho, P. Dimitriou et al., Nucl. Data Sheets 163, 109 (2020).
[3] International Atomic Energy Agency, IAEA Photonuclear Data Library.

[4] K. Mang'era, K. Ogbomo, R. Zriba, J. Fitzpatrick, J. Brown, E. Pellerin, J. Barnard, C. Saunders, M. de Jong, J. Radioanal. Nucl. Chem. 305, 79 (2015).

[5] K. Mang'era, Ph.D. Thesis, University of Manitoba, Canada 2014.

[6] C.K. Ross, W.T. Diamond, arXiv:1506.08065 (2015). 